\title{
Pulmonary artery anomalies: Interesting cases with a common embryonic basis
}

\author{
Badhrinarayanan $\mathrm{S}^{1}$, Venugopalan $\mathrm{P}^{2 *}$, and Pushparajah $\mathrm{K}^{3}$ \\ ${ }^{1}$ Medical Student, Brighton and Sussex Medical School, United Kingdom \\ ${ }^{2}$ Consultant Paediatrician, Brighton and Sussex University Hospitals NHS Trust, United Kingdom \\ ${ }^{3}$ Consultant Paediatric Cardiologist, Evelina London Children's Hospital, United Kingdom
}

\begin{abstract}
Isolated pulmonary artery anomalies are rare. We present two babies, one with absent left pulmonary artery (LPA) and the other with pulmonary artery sling (PA Sling), with similar clinical presentations. Both of these defects have a common embryonic origin.

We discuss the importance of a high index of clinical suspicion and clear delineation the left pulmonary artery during echocardiography to facilitate early diagnosis.
\end{abstract}

\section{Introduction}

The aortic arches or pharyngeal arch arteries (once referred to as branchial arches in human embryos) are a series of six paired embryological vascular structures which give rise to several major arteries (Figure 1). The branch pulmonary arteries arise from the proximal part of the sixth right arch and the proximal part of the sixth left arch. Defective development of the proximal portion of the sixth left arch gives rise to a disconnected left pulmonary artery or a pulmonary artery sling depending on where the distal left pulmonary artery gets its blood supply from [1-3].

Pulmonary artery anomalies can co-exist with other congenital heart defects, or can be an isolated anomaly.

\section{Methods}

Two babies who presented with unexplained desaturation were investigated. The results of investigation and management are

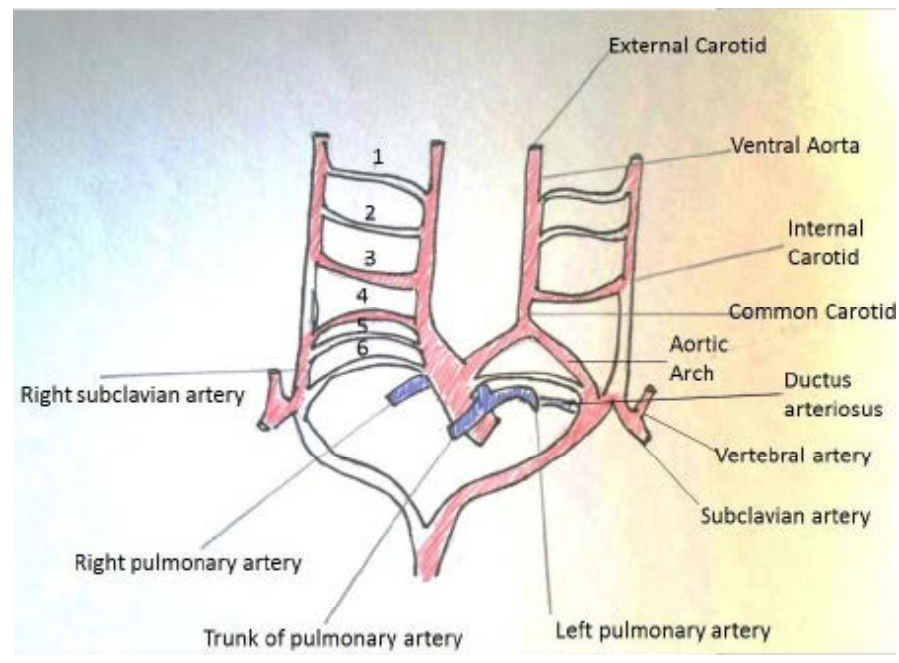

Figure 1. Diagram showing the primitive aortic arches and their evolution. reviewed. A Medline search using the keywords was performed and results summarized.

\section{Results}

\section{Case 1 - Absent/disconnected left pulmonary artery}

A term baby with $22 \mathrm{q}$ deletion presented on day 5 with shortness of breath, sweating during feeds and seizures (secondary to hypocalcaemia). Cardiovascular examination was normal except for a prominent systolic murmur. His oxygen saturation was $98 \%$ in all four limbs. Initial echocardiogram demonstrated a patent ductus arteriosus (PDA) and a foramen ovale (PFO).

In view of unexplained intermittent desaturations and a heart murmur, a repeat echocardiogram was organised, which documented in addition a right aortic arch, and absence of the left pulmonary artery. Cardiac MRI identified the disconnected LPA. At surgical exploration, a connection of the LPA to the left common carotid artery was identified through an atretic segment. A conduit was successfully constructed between the pulmonary trunk and the LPA, with relief to symptoms (Figure 2).

\section{Case 2 - Pulmonary artery sling}

A preterm 33 weeker exhibited shortness of breath, noisy breathing and blue episodes while feeding in the first few weeks of life. These

Correspondence to: Venugopalan P, Consultant Paediatrician with Cardiology Expertise \& Honorary Senior Clinical Lecturer, Departmental Lead for Cardiologys, Department of Paediatric Medicine, Level 6, Brighton \& Sussex University Hospitals NHS Trust, Level 6, Royal Alexandra Children's Hospital, Eastern Road, Brighton, BN2 5BE, United Kingdom, Tel: 01273 627008; Fax: 01273 627003; E-mail: p.venugopalan@bsuh.nhs.uk

Key words: disconnected left pulmonary artery, absent left pulmonary artery, pulmonary artery sling, vascular ring

Received: April 09, 2016; Accepted: May 02, 2016; Published: May 06, 2016 

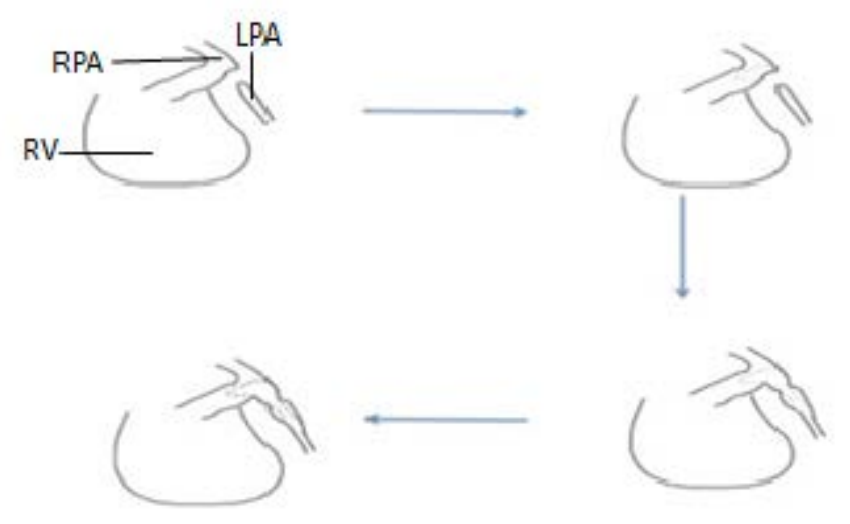

Figure 2. Diagram showing surgical reconstruction of disconnected left pulmonary artery (LPA) using a flap from the main pulmonary artery (RV: Right ventricle, RPA: Right pulmonary artery).
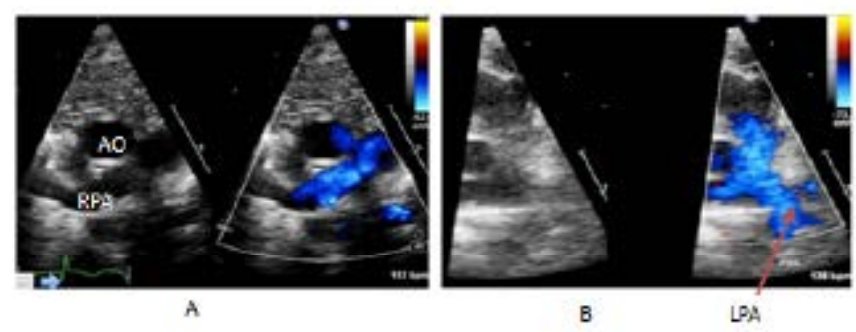

Figure 3. Left pulmonary artery (LPA) sling - pre-operative (A) and post-operative (B) echocardiograms (AO: Aorta, RPA: Right pulmonary artery).

symptoms persisted despite respiratory support with CPAP, treatment of sepsis and anti-reflux medication. An initial echocardiogram demonstrated a PDA and PFO. As symptoms persisted, a repeat echocardiogram demonstrated the distal origin of the LPA from the right pulmonary artery, that coursed round the origin of the left bronchus, with airway compromise.

Following surgical transection of the LPA at its origin and implantation into the main pulmonary artery, the baby achieved a good outcome with minimal respiratory symptoms (secondary to the bronchomalacia) (Figure 3 and 4).

\section{Discussion}

Both babies had pulmonary artery anomalies. Interesting, both these anomalies have a common embryonic origin related to the proximal segment of the primitive $6^{\text {th }}$ branchial arch arteries. Medline

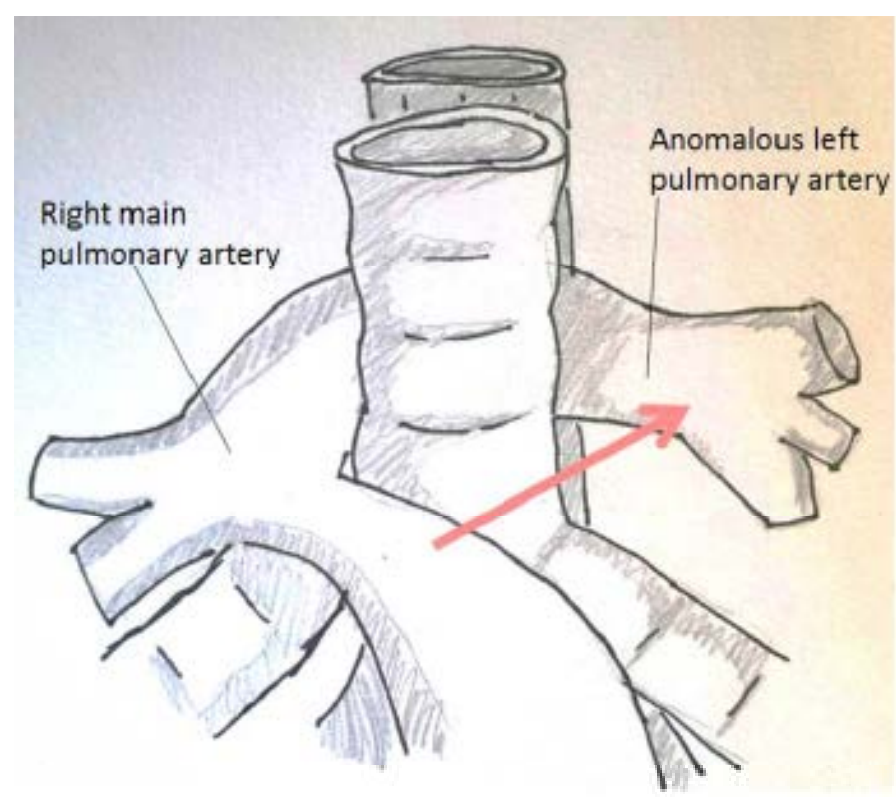

Figure 4. Diagram showing the diagnostic representation of the abnormal origin of the left pulmonary artery from the right pulmonary artery (RPA). The red arrow indicates the site where the left pulmonary artery normally originate from. The abnormal origin therefore creates a sling around the lower trachea/bronchus (LPA sling).

search emphasized the importance of early diagnosis is such situations, and highlighted the role of careful look at the branch pulmonary arteries during any echocardiogram. Early diagnosis and surgical recruitment facilitates growth of the pulmonary artery and optimizes associated lung development.

\section{Conclusion}

Anomalies of the pulmonary arteries have a common embryonic origin, and could be overlooked at initial echocardiograms. The pulmonary artery branches need careful attention during all neonatal echocardiograms. Once identified and repaired, outcome is good.

\section{References}

1. Bockeria LA, Makhachev OA, Khiriev TKh, Podzolkov VP, Zelenikin MA, et al. (2015) Repair of congenital heart defects associated with single pulmonary artery. Asian Cardiovasc Thorac Ann 23: 157-163. [Crossref]

2. Ntsinjana HN, Hughes ML, Taylor AM (2011) The role of cardiovascular magnetic resonance in pediatric congenital heart disease. J Cardiovasc Magn Reson 13: 51. [Crossref]

3. Barbero-Marcial M, Atik E, Baucia JA, Pradel HO, Macruz R, et al. (1988) Reconstruction of stenotic or nonconfluent pulmonary arteries simultaneously with a Blalock-Taussig shunt. J Thorac Cardiovasc Surg 95: 82-89. [Crossref]

Copyright: (C2016 Badhrinarayanan S. This is an open-access article distributed under the terms of the Creative Commons Attribution License, which permits unrestricted use, distribution, and reproduction in any medium, provided the original author and source are credited. 